\title{
$302 \mathrm{C}$
}

\section{Adoptée :}

LIGNE DIRECTRICE DE L'OCDE

12 mai 1981

POUR LES.ESSAIS DE PRODUITS CHIMIQUES Corrigé le 8 Septembre 2009

\section{«Biodégradabilité dite Intrinsèque : \\ Essai MITI Modifié (II) ».}

\section{INTRODUCTION}

\section{- Connaissances requises}

- Nécessité de disposer d'une méthóde d'analyse pour le dósage de la substance à examinèr dans la solution d'essai.

- Lá formule empirique de la substance à examiner est nécessaire pour le calcul de la demande théorique en oxygène (DTO):

\section{- Informations générales}

- Il sera utile de disposer d'informations sur les proportions relatives des principaux composants du produit à examiner lors de l'interprétation des résultats obtenus, en particulier dans lẹs cas où lẹs résultats se situent au voisinage de la limite.

- Il serait utile d'avoir des informations au sujet de la toxicité des produits chimiques pour pouvoir interpréter les valeựs faibles et pour sélectionner les concentrations d'essai appropriées.

\section{- Conditions particulières} l'essai:

La méthode ne s'applique qu'aux substances organiques qui, à la concentration utilisée dans

- ont une pression de vapeur négligeable,

- n'ont pas d'action inhibitrice sur les bactéries, et

- n'entrent pas en contact et ne réagissent pas avec l'absorbant de $\mathrm{CO}_{2}$.

Le Groupe d'Experts Dégradation/Accumulation de l'OCDE a estimé que cet essai convenait pour la détermination de la biodégradabilité dite intrinsèque des produits chimiques organiques dans des conditions aérobies. Il a fait l'objet d'un contrôle au cours du programme de comparaison en laboratoire de l'OCDE (1978 - 1980).

\section{- Recommandations}

Si la substance à examiner n'est pas soluble à la concentration d'essai, des dispositions particulières, telles que la dispersion par ultrasons, pourront être prises afin de parvenir à une dispersion satisfaisante de la substance à examiner. 


\section{- Documents de référence}

Cette Ligne directrice est basée sur un décret prescrivant l'essai des nouveaux produits chimiques, pris en application de lạ Loi sur la contrôle des substances chimiques (Loi no 117, 1973) (Décret du Premier Ministre du Japon, du Ministre de la Santé Publique et du Ministre du Commerce International et de l'Industrie, nọ 1 (13 juillet. 1974)).

\section{MÉTHODE}

\section{A. INTRODUCTION, OBJET, PORTÉE, PERTINENCE, APPLICATIONET LIMITES DE L'ESSAI}

Cet essai a pour but la mesure de la demande biochimique en oxygène (DBO) et l'analyse dẹs produits résiduels, afin d'évaluer la biodégradabilité dite intrinsèque des produits chimiques qui, d'après la méthodeMTI modifiée(I) possèdent un faible niveau de dégradabilité.

\section{- Définitions et unités}

ou

$$
\text { Pourcentage de dégradation }=\frac{D B O-B}{D T O} \times 100(\%)
$$

$$
\text { Pourcentage de dégradation }=\frac{S b-S a}{S b} \times 100(\%)
$$

DBO : Demande biologique en oxygène (expérimentale en mg) du composé à examiner, mesurée sur la courbe DBO

B : Consommation d'oxygène (expérimentale en $\mathrm{mg}$ ) d'un milieu de culture de base auquel est ajouté l'inoculum, mesurée sur la courbe DBO.

DTO : Demande théorique en oxygène (théorique en $\mathrm{mg}$ ) quand le composé ã examiner est complètement oxydé.

Sa : Quantité résiduelle (expérimentale en $\mathrm{mg}$ ) de composé à lissue de l'essai de biodégradabilité.

Sb : Quantité résiduelle (expérimentale en mg) de composé dans l'essai contrôle réalisé avec de l'eau à laquelle seul le composé à examiner a été ajouté. 


\section{« Biodégradabilité dite Intrinsèque : Essai MITI Modifié (II) 》}

\section{- Substance de référence}

Lors de l'étude d'une nouvelle substance, des substances de référence pourront être utiles dans certains cas, bien qu'il soit encore impossible de recọmmander des substances de référence particulières. Il est souhaitable d'utiliser des substances de contrôle pour vérifier l'activité de l'inoculum. A cet effet on utilisera de l'aniline, du benzoate de sodium ou de l'acétate de sodium.

Si le taux de dégradation de l'aniline, calculé à partir de la consommation d'oxygène, ne dépasse pas $40 \%$ au bout d'une semaine et $65 \%$ au bout de deux semaines, l'essai doit êtré considéré commie nul.

\section{- Principe de la méthode}

La méthode est fondée sur les principes suivants :

- les produits chimiques à tester représentent les seules sources de carbone organique.

- pas d'adaptation des micro-organismes sur les produits chimiques à examiner.

On utilise un appäreil de mesure automatisé de la consommation d'oxygène en circuit fermé (DBO-mètre). On inocule des micro-organismes dans des récipients contenant les produits chimiques à tester. Au cours de l'essai, la demande biochimique en oxygène est mesurée en continu au moyen d'un DBO-mètre. La biodégradabilité est calculée sur la base de la DBO et des analyses chimiques complémentaires, telles que la mesure de la concentration du carbone organique dissous, la concentration des produits résiduels etc.

\section{- Critères de qualité}

\section{Reproductibilité}

La reproductibilité de cette méthode est généralement bonne, spécialement dans le cas de produits chimiques solubles dans l'eau au-delà de $100 \mathrm{ppm}$.

\section{Sensibilité}

- Consommation d'oxygène: limite de détection $=1 \mathrm{mg}$ (consommation d'oxygène par les micro-organismes).

- Analyse chimique :

dépend de la sensibilité des méthodes analytiques utilisées. 


\section{$302 \mathrm{C}$}

page 4

$\therefore$
« Biodégradabilité dite Intrinsèque :

Essai MITI Modifié (II) »

\section{Spécificité}

Applicable à chaque type de produit chimique poư lequel $\mathrm{C}_{\text {ead }} / \mathrm{C}_{\mathrm{air}} \geq 1^{*}$.

Pour les produits chimiques volatils, utiliser un DBO-mètre** modifié.

\section{Possibilité de normalisation}

Cette méthode a été agréée par le Gouvernement Japonais sous le nom de. «Méthode d'essai de la biodégradabilité des substances chimiques par les micro-organismes »).

\section{Possibilité d'automatisation}

Lutilisation dun DBO-Mètre** permet l'enregistrement automatique de la consommation d'oxygène par les micro-organismes (en circuit fermẹ).

\section{B. MODE OPÉRATOIRE}

\section{- Préparations}

Appareillage : Un DBO-mètre équipé de six flacons de $300 \mathrm{ml}$.

Flacon $1 \quad$ eau désionisée***, $+300 \mathrm{ml}+$ produit à examiner, $9 \mathrm{mg}$

Flacon 2,3,4 : milieu de culture de base, $300 \mathrm{ml}+$ boue activée, $30 \mathrm{mg}$ (en poids $\mathrm{sec})+$ produit à examininer, $9 \mathrm{mg}$

Flacon 5 : milieu de culture de base, $300 \mathrm{ml}+$ boue activée, $9 \mathrm{mg}$ (en poids $\mathrm{sec})+$ aniline, $30 \mathrm{mg}$

Flacon $6 \quad:$ milieu de culture de base, $300 \mathrm{ml}+$ boue activée, $30 \mathrm{mg}$ (en poids sec):

\footnotetext{
* $\mathrm{C}=$ concentration

** Le.DBO-mètre modifié est constitué par un capillaire et un DBO-mètre normal. (Voir description à l'annexe 1).

*** L'eau utilisée ne doit pas contenir plus de 10 pour cent de carbone organique introduit par le produit à examiner.
} 


\section{« Biodégradabilité dite Intrinsèque : Essai MITI Modifié (II) »}

\section{Prétraitement du produit à examiner}

Si le composé à examiner n'est pas soluble dans l'eau à la concentration désirée, il devra être pulvérisé aussi finement que possible.

Si le composé à tester est volatil, il doit être refroidi correctement pour prévenir l'évaporation.

Le cas échéant, on procédera à lidentification du produit à examiner.

\section{Milieu de culture de base}

On prend $3 \mathrm{ml}$ de chacune des solutions $\mathrm{A}, \mathrm{B}, \mathrm{C}$, et $\mathrm{D}$, et l'on amène à $1000 \mathrm{ml}$ de l'eau. (On utilise dans tous les cas de l'eau désionisée).

Solution A : $\quad 21,75 \mathrm{~g}$ de phosphate dipotassique, $8,5 \mathrm{~g}$ de phosphate monopotassique, $44,6 \mathrm{~g}$ de phosphate disodique, $12 \mathrm{H}_{2} \mathrm{O}$ et $1,7 \mathrm{~g}$ de chlorure d'ammonium ; dissoudre dans l'eau et amener le volume à $1000 \mathrm{ml}$. (Le $\mathrm{pH}$ de la solution est de 7,2)

Solution B : $\quad 22,5 \mathrm{~g}$ de sulphate de magnésium $7 \mathrm{H}_{2} \mathrm{O}$; dissoudre dans l'eau et amener à $1000 \mathrm{ml}$.

Solution C: $\quad 27,5 \mathrm{~g}$ de chlorure de calcium ; dissoudre dans l'eau et amener à $1000 \mathrm{ml}$.

Solution D : $\quad 0,25 \mathrm{~g}$ de chlorure ferrique $6 \mathrm{H}_{2} \mathrm{O}$; dissoudre dans l'eau et amener à $1000 \mathrm{ml}$.

\section{Boue activée}

Point d'échantillonnage des boues : Les boues sont prélevées, en principe, dans au moins dix endroits, répartis dans l'ensemble du pays, en particulier dans les zones où la consommation et le rejet de divers types de substances chimiques sont importants.

C'est ainsi que la boue activée normalisée du Centre Japonais de «Biotesting Chimique » est un mélange de prélèvements effectués dans les emplacements suivants :

- Installations de traitement des eaux usées urbaines : 3 installations localisées au nord, au centre et au sud du Japon.

- Installation de traitement des eaux usées industrielles: une installation utilisée pour le traitement des eaux usées d'usines chimiques. 


\section{Essai MITI Modifié (II) »}

- Rivière : 3 rivières situées au nord, au centre et au sud du Japon.

- Lac : un lac situé au centre du Japon.

-- Mer : 2 mers intérieures du Japon.

Fréquence d'échantillonnage des boues : Les boues doivent, en principe, être prélevées quatre fois par an, en mars, juin, septembre et décembre.

\section{Méthodes d'échantillonnage des boues}

- Eaux usées urbaines : 1 litre de boue recirculée dans une installation de traitement des eaux. usées.

- Rivières, lacs et marais ou mer : 1 litre d'eau de surface et 1 litre de sol prélevé à la surface de la plage en contact avec l'atmosphère.

Préparation : Les échantillons de boue prélevés aux points d'échantillonnage sont mélangés par agitation dans un seul récipient et on laisse reposer le mélange. Les substances étrangères qui flottent à la surface sont éliminées et l'on filtre le surnageant à l'aide de papier filtre $n^{\circ} 2$. Le filtrat est ajusté à $\mathrm{pH} 7 \pm 1.0$ par addition de soude ou d'acide phosphorique et transféré dans un bac de culture et aéré.

Culture : 30 minutes environ après avoir arrêté l'aération de la solution obtenue, on élimine environ $1 / 3$ du volume total du surnageant: On ajoute un volume égal d'une eau usée synthétique à $0,1 \% *$ aux $2 / 3$ restant du surnageant et le mélange est aéré de nouveau. L'opération est répétée une fois par jour. La culture est réalisée à $25 \pm 2{ }^{\circ} \mathrm{C}$.

Contrôle : Pour contrôler l'étape de culture, on vérifie les points suivants et on procède aux ajustements nécessaires :

- Aspect du surnageant : le surnageant de la boue activée doit être clair.

- Précipitabilité des boues activées : les boues activées, qui se présentent sous l'aspect de larges flocons, doivent avoir une capacité de précipitation élevée.

- Etat de la boue activée : si l'on n'observe pas de formation de flocons, on augmente soit le volume d'eau usée synthétique à $0,1 \%$, soit la fréquence d'addition de l'eau usée synthétique.

* Eau usée synthétique à $0,1 \%$ : on dissout dans un litre d'eau $1 \mathrm{~g}$ de glucose, $1 \mathrm{~g}$ de peptones et $1 \mathrm{~g}$ de phosphate monopotassique et on ajuste la solution à $\mathrm{pH} 7.0 \pm 1.0$ par addition de soude. 


\section{« Biodégradabilité dite Intrinsèque :} Essai MITI Modifié (II) »

$-\mathrm{pH}:$ le $\mathrm{pH}$ du surnageant est de $7.0 \pm 1 ; 0$

- Température : la température de culture d'une boue activée est de $25^{\circ} \mathrm{C} \pm 2{ }^{\circ} \mathrm{C}$.

- Aération nécessaire : lors du remplacement du surnageant par l'eau usée synthétique, la solution contenue dans le bac de culture doit être suffisamment aérée pour que la concentration en oxygène dissous de la solution se maintienne au-dessus de $5 \mathrm{ppm}$.

- Microflore de la boue activée : lorsqu'on observe la boue activée au microscope (grossissement $x 100 \sim 400$ ), on doit voir différentes espèces de protozoaires qui accompagnent les flocons.

- Mélange de boues activées fraîches et anciennes : pour maintenir au même niveau d'activité les boues fraîches et les anciennes, on mélange le filtrat du liquide surnageant d'une boue activée utilisée effectivement dans l'essai avec un volume égal de filtrat du surnageant d'une boue activée fraîchement collectée et le mélange est mis en culture.

- Vérification de l'activité d'une boue activée : on vérifiera périodiquement (au moins une fois tous les trois mois) l'activité de la boue activée au moyen de substances étalons en appliquant la méthode indiquée ci-dessous. En cas de mélange d'échantillons de boues activées fraîches et anciennes, on procèdera à une vérification soigneuse par rapport à la boue activée ancienne.

\section{Exemple de préparation d'échantillons de boue activée et période d'utilisation.}

Déc. Janv. Févr.

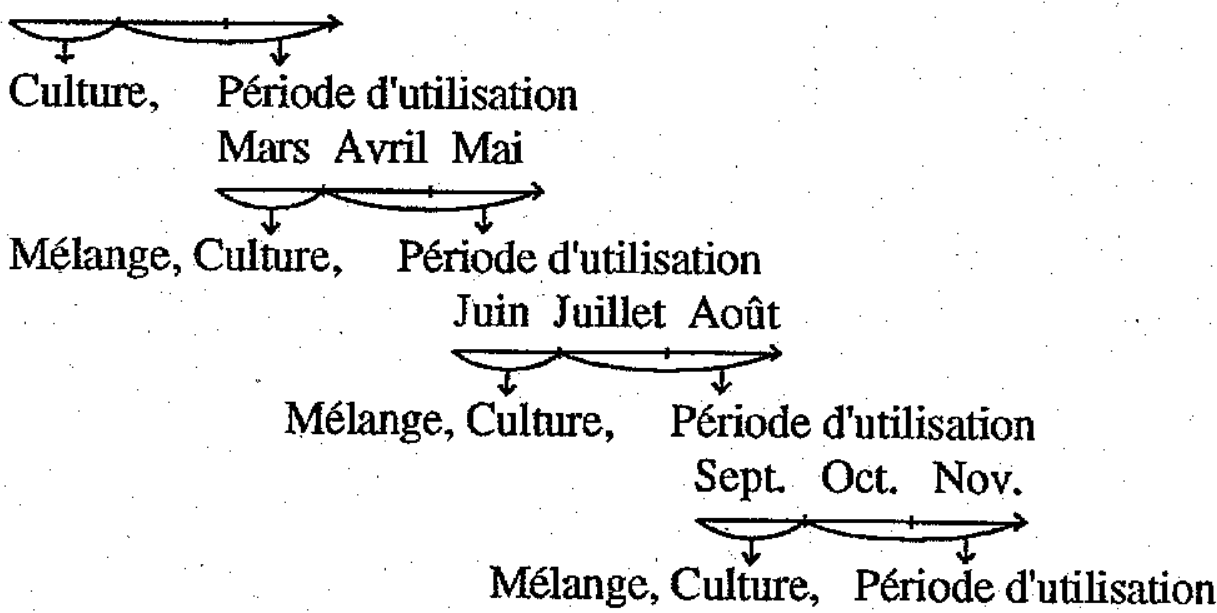

(Le même schéma de préparation et d'utilisation est répété). 


\section{$302 \mathrm{C}$}

page 8

\section{« Biodégradabilité dite Intrinsèque :}

Essai MITI Modifié (II) »

\section{Addition du composé à examiner et préparation de l'essai}

On utilise et on porte à la température d'essai les récipients suivants :

(1) Un récipient contenant le milieu de culture de base, auquel on ajoute $30 \mathrm{ppm}$ (poids/volume) du composé à examiner; on ajuste le $\mathrm{pH}$ de cette solution à 7 avant d'inoculer la boue activée, si nécessaire.

(2) Un récipient pour le contrôle, contenant uniquement le milieu de culture de base.

(3) Un récipient contenant de l'eau à laquelle on ajoute $30 \mathrm{ppm}$ (poids/volume) du composé à examiner.

(4) Un récipient contenant le milieu de culture de base auquel on ajoute $100 \mathrm{ppm}$ (poids/volume) d'aniline ou d'autre substance de contrôle.

\section{Ensemencement de la boue activée}

On ajoute linoculum aux récipients 1) et 2) ci-dessus de manière à ce que la concentration de matière en suspension $(100 \mathrm{ppm}[\mathrm{v} / \mathrm{v}])$ requise dans la Norme Industrielle Japonaise décrite à l'annexe 2 soit atteinte.

Dans le cas du récipient 4 , la concentration de matière en suspension est de $30 \mathrm{ppm}$.

\section{- Conditions expérimentales}

- Concentration des produits à examiner : $30 \mathrm{ppm}$ (poids/volume)

- Concentration de la boue activée : $100 \mathrm{ppm}$ (poids/volume)

- Température $: 25^{\circ} \mathrm{C} \pm 2{ }^{\circ} \mathrm{C}$

- Période : de 14 à 28 jours

- Procéder dans l'obscurité. La température et le changement de couleur du contenu du bac de culture doivent être vérifiés tous les jours.

- Agiter énergiquement avec un agitateur mécanique.

- Exécution de l'essai

La courbe DBO est obtenue automatiquement et en continu pendant une période de 14 à 28 jours (voir figure 1). 


\section{«Biodégradabilité dite Intrinsèque :}

\section{Essai MITI Modifié (II) »}

Figure $1:$ Courbe de dégradation d'un composé facilement biodégradable.

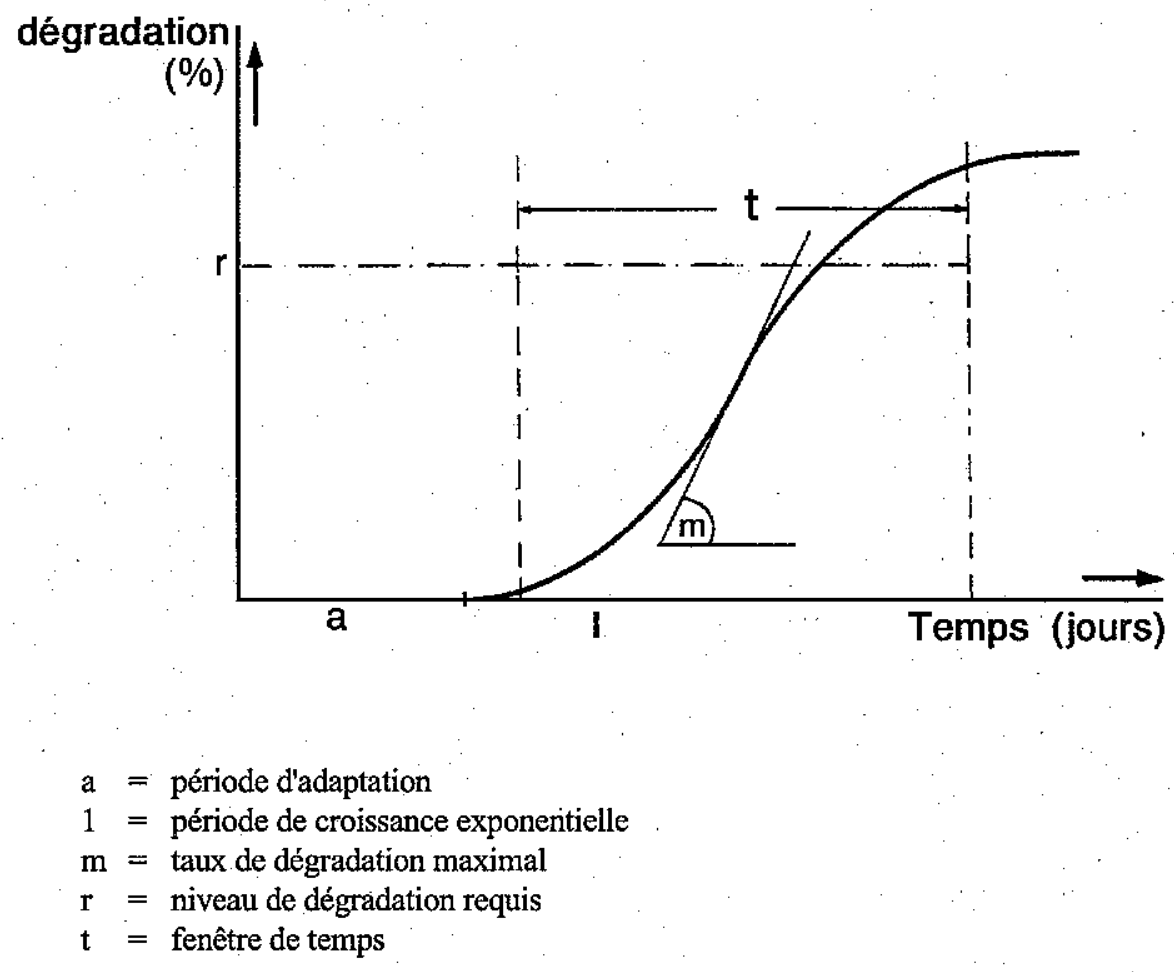

Au bout de la période d'essai de 14 à 28 jours, on analyse le $\mathrm{pH}$, les produits résiduels et les produits intermédiaires dans les récipients. On analyse également les produits à examiner dans le récipient qui ne contient pas de boue activée afin de déterminer s'il s'est produit un changement quelconque du produit au cours de la période d'essai ou une déperdition quelconque du produit original par suite d'évaporation ou d'adsorption par les parois des récipients, etc.

\section{- Moyens analytiques}

Si le composé à examiner est soluble dans l'eau, on dose également la quantité résiduelle du carbone organique total.

(a) Utilisation d'un analyseur de carbone organique total : on prélève du récipient d'essai $10 \mathrm{ml}$ de la solution à examiner et on centrifuge à $3000 \mathrm{~g}$ pendant cinq minutes. La quantité résiduelle de carbone organique total dans le liquide surnageant est dosée sur un analyseur de carbone organique total. 
page 10

\section{« Biodégradabilité dite Intrinsèque : Essai MITI Modifié (II) »}

(b) Utilisation d'autres analyseur's : extraire, au moyen d'un solvant approprié pour le composé à analyser, le contenu total d'un récipient d'essai et, après un traitement approprié, (concentration par exemple, doser la quantité résiduelle du composé sur un appareil analyseur (chromatographie en phase gazeuse, spectrométrie d'absorption; spectrométrie de masse, spectrophotométrie d'absorption atomique, etc.).

Dans le cas de produits chimiques volatils, le bain de contrôle de température du DBOmètre sera refroidi à $10^{\circ} \mathrm{C}$ et cette température sera maintenue pendant au moins $30 \mathrm{~min}$, afin d'éviter l'évaporation. Les méthodes analytiques (a) et (b) seront alors appliquées.

\section{RESULTATS ET RAPPORT}

\section{- Calculdes résultats}

a) Méthode de calcul du pourcentage de dégradation à partir de la consommation d'oxygène :

$$
\text { Pourcentage de dégradation }=\frac{D B O-B}{D T O} \times 100(\%)
$$

DBO : Demande biologique en oxygène (expérimental en $\mathrm{mg}$ ) du composé à examiner, mesurée sur la courbe DBO.

B : : Consommation d'oxygène (expérimental en $\mathrm{mg}$ ) d'un milieu de culture de base auquel est ajouté l'inoculum, mesurée sur la courbe DBO.

DTO : Demande théorique en oxygène (théorique en $\mathrm{mg}$ ) nécessaire quand le composé à examiner est complètement oxydé.

b) Méthode de calcul du taux de dégradation à partir du résultat de l'analyse directe :

$$
\text { Taux de dégradation }=\frac{S b-S a}{S b} \times 100(\%)
$$

$\mathrm{Sa} \quad$ : Quantité résiduelle (expérimental en $\mathrm{mg}$ ) de composé à l'issue de l'essai de biodégradabilité.

$\mathrm{Sb} \quad$ : Quantité résiduelle moyenne (expérimental en $\mathrm{mg}$ ) de composé dans les deux essais contrôles réalisés avec de l'eau à laquelle seul le composé à examiner a été ajouté. 


\section{« Biodégradabilité dite Intrinsèque :} Essai MITI Modifié (II) »

- Evaluation des résultats

- Calcul de la demande théorique en oxygène

$\begin{array}{ll}\text { Elément } & \text { Forme oxydé } \\ \mathrm{C} & \mathrm{CO}_{2} \\ \mathrm{H} & \mathrm{H}_{2} \mathrm{O} \\ \mathrm{N} & \mathrm{NO}_{2} \\ \mathrm{~S} & \mathrm{SO}_{2} \\ \mathrm{X} \text { (halogène) } & \mathrm{X}\end{array}$

- Taux de récupération de la méthode analytique.

- Rapport

Le rapport doit comprendre les points suivants :

- Informations sur les produits à examiner

Nom, formule structurale, poids moléculaire, degré đe pureté, types d'impuretés, propriétés physico-chimiques du produit à examiner, données relatives à l'interprétation du spectre du produit à examiner.

- Conditions de l'essai

Boue activée : : point de prélèvement et concentration de la boue.

Produit à examiner : concentration

Période d'essai

Température d'essai

- Méthode analytique

Prétraitement

Etat des appareils analytiques

Taux de récupération de l'analyse

Identification des produits intermédiaires

- Résultats

Courbe $\mathrm{DBO}$ et dénomination de l'appareil

$\mathrm{DBO}$ (m)

B (mg)

$\mathrm{Sa} \quad(\mathrm{mg})$

$\mathrm{Sb} \quad(\mathrm{mg})$

DTO (mg)

Taux de dégradation déterminé à partir de la DBO

Taux de dégradation déterminé par analyse chimique

Chromatogrammes ou spectres des produits chimiques obtenus et utilisés aux fins de l'analyse.

- Remarques 


\section{$302 \mathrm{C}$}

page 12

\section{« Biodégradabilité dite Intrinsèque : Essai MITI Modifié (II) »}

\section{- Interprétation des résultats}

Pour établir une comparaison avec les composés de référence, la biodégradabilité de la substance à examiner est classée par rapport à la dégradabilité de l'aniline.

Si le taux de dégradation de l'aniline calculé à partir de la consommation d'oxygène n'est pas supérieur à $40 \%$ au bout d'une semaine et de $65 \%$ au bout de deux semaines, l'essai doit être considéré comme nul. Si le taux de récupération de $\mathrm{Sb}$ se révèle de l'ordre de $10 \%$ au moins, l'essai est également considéré comme nul.

Dans ces conditions, la variation de la consommation d'oxygène de base peut être tellement élevée par rapport aux conditions standard que l'évaluation du produit à examiner en cas d'utilisation de la valeur DBO, doit être menée avec soin. (Comme la concentration du produit à examiner est plus faible, la valeur DBO absolue peut être rélativement plus basse que la valeur DBO dans les conditions normales).

\section{B I B L I O G R A P H I E}

1. Biodegradability and bio-accumulation test of chemical substances (C-5/98/JAP), 1978.

2. The chemical substances control law in Japan (Chemical Products Safety Division, Basic Industries Bureau, MITI) (C-2/78/JAP), 1978.

3. The biodegradability and bio-accumulation of new and existing chemical substances 5, 8 (C3/78/Jap) 1978. 


\section{« Biodégradabilité dite Intrinsèque : Essai MITI Modifié (II) 》

\section{AN N X E}

\section{PRINCIPE DE FONCTIONNEMENT DE L'APPAREIL ¿ MESURER LA CONSOMMATION D'OXYGÈNE EN CIRCUIT FERMÉ}

Le coulombmètre est un instrument destiné à mesurer électrochimiquement l'oxygène consommé par les micro-organismes. (Coulombmétrie).

On trouvera ci-dessous le schéma de fonctionnement de l'appareil. (Dans l'appareil à mesurer la DBO modifiée* les parties hachurées des tubes sont à remplacer par des tubes capillaires).

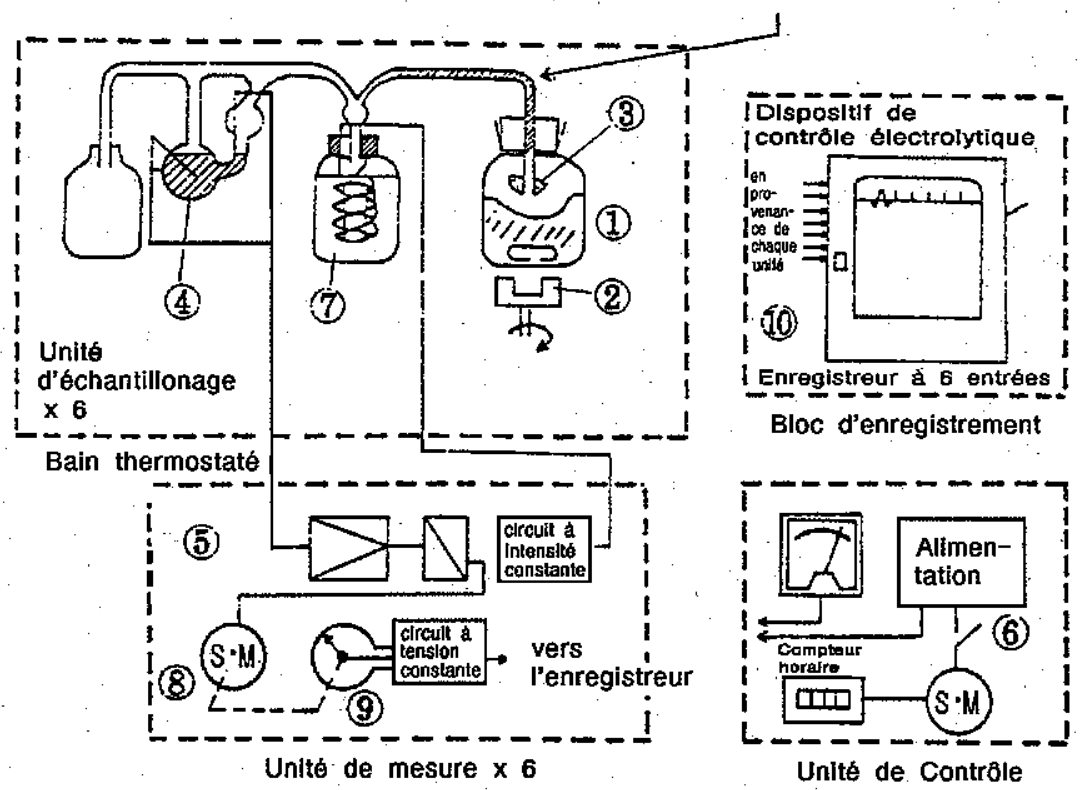

L'échantillon contenu dans le flacon d'incubation (1) est agité au moyen d'un agitateur magnétique (2). A mesure que la réaction progresse, l'oxygène dissous dans le liquide est consommé. L'oxygène $\left(\mathrm{O}_{2}\right)$ contenu dans la partie vide du flacon d'incubation est dissous dans le liquide, ce qui donne lieu à un dégagement de $\mathrm{CO}_{2}$.

Le $\mathrm{CO}_{2}$ étant absorbé par la chaux sodée 3 , la pression partielle d'oxygène dans la partie vide; ainsi que la pression totale diminuent.

* L'appareil à mesurer la DBO est fabriqué par Ohkura Electric Co., Ltd. 1-11-16, Shibuya, Shibuya-ku, Tokyo, Japan. (Pour les produits chimiques volatils, installer un tube capillaire entre chaque récipient d'essai et chaque flacon d'électrolyse). 


\section{$302 \mathrm{C}$}

page 14

\section{« Biodégradabilité dite Intrinsèque : Essai MITI Modifié (II) »}

La baisse de pression est détectée et convertie en signal électrique par un manomètre à électrode (4), tandis que le signal est amplifié par l'amplificateur (5) pour actionner le relais (6) ce qui provoque la mise en marche du moteur synchrone (8). Simultanément, sous l'action d'un courant à intensité constante, de l'oxygène est produit par l'électrolyse d'une solution de cuivre dans de l'acide sulfurique contenue dans le flacon d'électrolyse (7).

Cet oxygène est envoyé dans le flacon d'incubation et la remontée de la pression résultante est détectée par le manomètre, ce qui a pour effet de couper le relais et d'arrêter l'électrolyse et le moteur synchrone.

L'espace situé au-dessus du liquide dans le flacon d'incubation est maintenu à pression constante en oxygène, et la consommation d'oxygène dans le flacon d'incubation est proportionnelle à l'oxygène produit par électrolyse. Cette dernière quantité étant proportionnelle à la durée d'électrolyse, il en résulte un courant électrolytique constant. En conséquence, l'angle de rotation du moteur synchrone (9) est transformé en signal $\mathrm{mV}$ au moyen d'un potentiomètre d'interverrouillage, ce qui se traduit par une indication de la quantité d'oxygène consommée sur l'enregistreur (10).

\section{MATIÈRES EN SUSPENSION}

(Normes industrielles japonaises K0102 - 10.2)

Les matières en suspension sont celles qui peuvent être séparées par filtration ou par centrifugation. Leur quantité peut être déterminée par une des méthodes exposées ci-après. En cas de difficulté à filttrer l'eau à examiner, procéder par centrifugation. Si l'eau contient énormément de matières en suspension, utiliser un filtre Büchner.

L'eau à examiner est prélevée dans de l'eau d'égout passée dans un tamis aux mailles de $2 \mathrm{~mm}$. Une quantité de $5 \mathrm{mg}$ au moins de filtrat est nécessaire pour procéder à la détermination. 


\section{« Biodégradabilité dite Intrinsèque :} Essai MITI Modifié (II) »

\section{A. FILTRATION SUR PAPIER FILTRE}

\section{- Méthode utilisant un filtre en verre fritté}

\section{Appareillage}

Filtre en verre fritté : Utiliser un creuset filtrant pourvu d'une plaque en verre fritté $1 \mathrm{G} 2$ ou un filtre Büchner du type 3G2.

\section{Mode opératoire}

Préparer deux filtres en verre fritté de même type et approximativement de même poids ; y déposer six feuilles de papier filtre et verser de l'eau dessus à plusieurs reprises pour les faire adhérer par succion. Placer ensuite les filtres dans une étuve à air chaud et les sécher pendant deux heures à une température de 105 à $110^{\circ} \mathrm{C}$.

Les refroidir dans un dessicateur et les peser (en cas d'utilisation d'une balance analytique le filtre le plus léger peut être utilisé comme poids supplémentaire). Verser ensuite une quantité appropriée d'eau à examiner sur le filtre le plus lourd"*, filtrer par aspiration, puis, à l'aide du filtrat, laver la paroi du filtre afin de la débarrasser des substances adhérentes (répéter l'opération si nécessaire). Verser ensuite le filtrat à plusieurs reprises sur le filtre le plus léger et filtrer par aspiration. Sécher les deux filtres dans l'étuve à air chaud pendant 2 heures à une température de 105 à $110^{\circ} \mathrm{C}$, puis laisser refroidir dans le dessicateur. Peser chaque filtre (en cas d'utilisation d'une balance analytique, le filtre le plus léger peut être utilisé comme poids supplémentaire), déterminer la différence de poids avant et après filtration et calculer la quantité de matière en suspension en ppm, selon la formule ci-après :

$$
S=(a-b) \times \frac{1000}{V}
$$

* Prendre une quantité d'eau à examiner qui permet d'obtenir après séchage pas plus de $5 \mathrm{mg}$ de matière en suspension (200 ml suffisent généralement). Toutefois, si l'eau d'essai est difficile à filtrer, ajouter $10 \mathrm{ml}$ de chaque échantillon d'eau d'essai en cours de filtration, en provenance des $10 \mathrm{ml}$ du cylindre de mesure pendant la filtration. 
Dans cette formule :

S: est la matière en suspension (ppm)

a : est la différence de poids avant et après filtration de l'eau d'essai (mg)

$\mathrm{b}$ : est la différence de poids avant et après filtration du filtrat (mg) (lors de l'utilisation d'une balance analytique, $b=0$ )

$\mathrm{v}$ : est la quantité d'eau d'essai $(\mathrm{ml})$.

\section{Remarques}

(1) Lorsqu'il s'agit de déterminer la perte par combustion des matières volatiles en suspension, effectuer l'essai selon la méthode décrite ci-dessous (3) (filtration sur filtre en fibre de verre).

On peut aussi procéder en transvasant la matière en suspension et le filtre dans un creuset ou un récipient d'évaporation, et procéder ensuite au séchage et à la combustion dans un four à moufle.

(2) Si le résidu de la solution évaporée est inférieur à $5000 \mathrm{ppm}$, on peut omettre la correction due à la différence en poids du filtrat avant et après filtration. Toutefois, en cas d'emploi d'une balance analytique, utiliser le filtre le plus léger comme poids supplémentaire, de sorte que la filtration du filtrat puisse être effectuée en même temps.

Même lorsqu'on utilise une balance à lecture directe, le poids varie suivant les propriétés hygroscopiques des substances contenues dans l'eau à examiner et suivant d'autres facteurs. Il est donc souhaitable d'effectuer la correction sur la base de la valeur obtenue par un essai à blanc avec le filtre à travers lequel on aura fait passer le filtrat. Lorsque l'eau à examiner contient des graisses, des huiles, des cires etc., une partie de ces substances sont déterminées comme de la matière en suspension.

Lorsqu'il s'agit de déterminer les matières en suspension, à l'exclusion des huiles et des graisses, verser plusieurs doses successives de $10 \mathrm{ml}$ de n-hexane dans le filtre préalablement séché et pesé après filtrage pour enlever les graisses et les huiles. Sécher ensuite le filtre et peser.

(3) Filtration sur filtre en fibre de verre (méthode GFP) : Fixer sur un support approprié un filtre de verre* de poids connu, (préalablement séché à $105-110^{\circ} \mathrm{C}$ pendant 2 heures après lavage).

* Whatman GF/B ou équivalent. 


\section{« Biodégradabilité dite Intrinsèque : Essai MITI Modifié (II) »

Verser une quantité suffisante d'eau à examiner pour obtenir, après séchage, plus de $5 \mathrm{mg}$ de matières en suspension. Après filtration par aspiration, reverser une partie du filtrat dans le récipient d'origine contenant l'eau à examiner. Laver les parois du récipient de manière à les débarrasser des matières en suspension adhérentes et les refiltrer sur le filtre en fibre de verre par aspiration. Répéter l'opération plusieurs fois. Enlever ensuite le filtre et le poser sur un verre à eau. Opérer alors selon la méthode de Büchner décrite ci-dessous de manière à déterminer la teneur en matières en suspension.

Après avoir déterminé les matières en suspension, évaluer leur résidu de combustion, le cas échéant conformément au mode opératoire décrit ci-dessous (filtration sur couche d'amiante).

\section{- Utilisation d'un filtre Büchner}

Cette méthode s'applique aux échantillons contenant une grande quantité de matières en suspension (par exemple de la boue)

\section{Appareillage}

Plaque perforée : en acier inoxydable (SUS 27 ou 28); épaisseur environ 0,5 mm; diamètre $50 \mathrm{~mm}$ ou $90 \mathrm{~mm}$. La plaque doit avoir la forme d'un verre de montre aux bords légèrement relevés. Toutes la surface plane est percée de trous de $0,5 \mathrm{~mm}$ de diamètre, disposés à intervalles réguliers.

Joint en caoutchouc : Le joint en caoutchouc consiste en un anneau de 2 à $3 \mathrm{~mm}$ d'épaisseur, d'une largeur d'environ $10 \mathrm{~mm}$ et d'un diamètre compris entre 10 et $90 \mathrm{~mm}$. Il peut entrer dans un filtre Büchner et peut être utilisé pour la filtration par aspiration, la plaque perforée étant posée dessus.

Filtre Büchner : Diamètre de $50 \mathrm{~mm}$ ou $90 \mathrm{~mm}$.

\section{Mode opératoire}

Préparer deux plaques perforées. Introduire le joint en caoutchouc dans le filtre Büchner et placer la plaque perforée dessus. Déposer ensuite le papier filtre ( $\left.\mathrm{n}^{\circ} 6\right)$, passer de l'eau plusieurs fois sur ce dernier et aspirer. Enlever le papier filtre en même temps que la plaque perforée et 


\section{$302 \mathrm{C}$}

page 18

\section{« Biodégradabilité dite Intrinsèque :} Essai MITI Modifié (II) »

sécher à $105-110^{\circ} \mathrm{C}$ pendant deux à trois heures. Laisser refroidir dans un dessicateur et peser à poids constant (lors de l'utilisation d'une balance analytique, la plus légère plaque perforée peut servir de poids supplémentaire).

Placer ensuite dans le filtre la plaque perforée la plus lourde avec son papier filtre. Filtrer 200 à $400 \mathrm{ml}$ d'eau à examiner par aspiration. Passer ensuite à plusieurs reprises le filtrat sur la plaque perforée plus légère munie de son papier filtre, et procéder comme dans le cas de la première plaque.

Déterminer la différence de poids avant et après l'opération. Calculer la quantité de matières en suspension (en ppm) dans l'eau à examiner au moyen de la formule suivante :

$$
S=(a-b) \times \frac{1000}{V}
$$

Dans cette formule :

$\mathrm{S}$ : est la matière en suspension (ppm)

a : est la différence entre le poids avant la filtration et le poids après la filtration de l'eau à examiner (en mg)

$\mathrm{b}$ : est la différence de poids avant et après filtration du filtrat (en $\mathrm{mg}$ ) (lorsqu'on utilise une balance analytique, $\mathrm{b}=0$ )

$\mathrm{v}$ : est l'eau à examiner (en $\mathrm{ml})$.

Voir remarques 1 à 3 ci-dessus.

\section{B. FILTRATION SUR COUCHE D'AMIANTE}

\section{Appareillage}

Creuset Gooch de 25 à $35 \mathrm{ml}$.

\section{Réactifs}

Suspension d'amiante : ajouter de l'eau à $15 \mathrm{~g}$ d'amiante et, après élimination des particules fines par décantations successives, compléter avec de l'eau jusqu'à 11 . 


\section{« Biodégradabilité dite Intrinsèque :} Essai MITI Modifié (II) »

\section{Mode opératoire}

Préparer deux creusets Gooch de même forme et approximativement de même poids. Après séchage, verser environ $20 \mathrm{ml}$ de l'amiante en suspension bien agitée, de manière à obtenir une couche d'amiante d'environ $3 \mathrm{~mm}$ d'épaisseur (environ $0,3 \mathrm{~g}$ )* et aspirer légèrement.

Placer ensuite les creusets dans une étuve à air chaud. Après séchage pendant deux heures à $105-110^{\circ} \mathrm{C}$, laisser refroidir dans un dessicateur jusqu'à un poids constant et mesurer le poids de chaque creuset (lors de l'utilisation d'une balance analytique, utiliser le plus léger des creusets comme poids supplémentaire). Fixer le plus lourd des creusets à la fiole à vide et verser dans le creuset assez d'eau à examiner pour obtenir $5 \mathrm{mg}$ de matières en suspension après séchage, puis filtrer doucement par aspiration. Répéter alors la filtration de la portion initiale de filtrat.

Tout en aspirant, verser à plusieurs reprises une petite quantité de filtrat dans. le creuset le plus léger, puis sécher dans une étuve à air chaud pendant deux heures à $105-110^{\circ} \mathrm{C}$; laisser refroidir dans un dessicateur. Peser le creuset et déterminer la différence de poids en utilisant le creuset comme poids supplémentaire, puis calculer le ppm de matières en suspension au moyen de la formule suivante :

$$
S=(a-b) \times \frac{1000}{V}
$$

(voir plus haut pour l'explication des symboles).

Remarque : L'eau à examiner doit être échantillonnée comme décrit dans la méthode utilisant le filtre en fibre de verre. Lorsque la quantité de résidus volatils solubles est inférieure à $5000 \mathrm{ppm}$, se reporter à la remarque 2 relative à la même méthode.

\section{MÉTHODE DE CENTRIFUGATION}

Cette mẹthode s'applique aux échantillons contenant des matières en suspension très difficiles à filtrer.

Appareillage

Centrifugeuse tournant à environ $2000 \mathrm{tr} / \mathrm{mn}$. Tube à précipitation de 50 à $100 \mathrm{ml}$.

* Après avoir versé la moitié de la suspension d'amiante, mettre en place la plaque perforée et verser le reste de la solution, 

Essai MITI Modifié (II) »

\section{Mode opératoire}

Verser dans le tube à précipitation assez d'eau à examiner pour obtenir plus de $5 \mathrm{mg}$ de matières en suspension.

Après avoir pesé chaque tube, centrifuger à environ $2000 \mathrm{tr} / \mathrm{mn}$ pendant 20 minutes, pour faire précipiter la matière en suspension. Eliminer par décantation* le liquide surnageant.

Ajouter au précipité $10 \mathrm{ml}$ d'eau à examiner, recommencer là centrifugation et éliminer par décantation le liquide surnageant.

Transférer le précipité dans un récipient d'évaporation préalablement chauffé à $105-110^{\circ} \mathrm{C}$ jusqu'à poids constant, puis évaporer à sec dans un bain à ébullition. Après séchage à l'étuve à 105 $110^{\circ} \mathrm{C}$ pendant 2 heures, laisser refroidir dans un dessicateur et peser. En cas d'utilisation d'une balance analytique, employer comme poids supplémentaire un récipient d'évaporation de même forme après avoir effectué l'essai à blanc.

Déterminer ensuite la différence entre le poids avant l'opération et le poids après l'opération. Calculer le ppm de matières en suspension au moyen de la formule ci-après :

$$
S=a \times \frac{1000}{V}
$$

(voir plus haut l'explication des symboles).

Remarque : Pour la méthode de séparation par centrifugation, il faut que la phase dispersée et le milieu de dispersion possèdent une densité différente. Lorsqu'une particule de $1 \mathrm{mg}$ située à $\mathrm{r} \mathrm{cm}$ du centre de rotation est centrifugée à une vitesse angulaire de $\mathrm{w} \mathrm{rad} / \mathrm{s}$, elle est soumise à la force centrifuge suivante :

en supposant que la masse de milieu de dispersion rejeté par une particule soit $1 \mathrm{mg}$.

$$
F=\left(m-m^{\prime}\right) w^{2} r
$$

on a :

soit RCF la force centrifuge spécifique et $\mathrm{N}$ (tours/minute) la vitesse de rotation, on obtient :

* Lorsqu'il s'agit de déterminer consécutivement les résidus solubles évaporés, garder le liquide surnageant. 


$$
R F C=\frac{F}{\left(m-m^{\prime}\right) g}=\frac{w^{2} r}{g}=0,00001118 r N^{2}
$$

Cette équation montre que la force centrifuge est différente à la surface et au fond du liquide. Par exemple si $\mathrm{N}=2000 \mathrm{tr} / \mathrm{mn}$ et $\mathrm{r}=5 \mathrm{~cm}$ (distance entre la surface du liquide dans le tube à précipitation et le centre de rotation), RCF est égal à $223 \mathrm{~g}$; lorsque $\mathrm{r}=13 \mathrm{~cm}$, RCF devient $581 \mathrm{~g}$. On notera donc la valeur de RCF près de la surface et au fond du tube.

Profondeur de la couche de liquide $=$

$$
\frac{(R C F \text { au fond })-(R C F \text { à la surface })}{(R C F \text { au fond })} \times(\text { distance jusqu'au fond })
$$

Dans cette méthode, on se base sur une centrifugeuse tournant à $2000 \mathrm{tr} / \mathrm{mn}$ dont le fond est à $13 \mathrm{~cm}$ de l'axe de rotation.

- Calcul de la quantité de matières en

suspension à partir de la différence en poids
de résidus d'évaporation

Pour calculer la quantité de matière en suspension à partir de la différence entre le poids . sec total du résidu et le résidu sec soluble, on applique la formule suivante :

$$
\begin{aligned}
& A=B-C \\
& \text { Dans cette formule : } \\
& A=\text { est la matière en suspension (ppm) } \\
& B=\text { est le poids sec total du résidu (ppm) } \\
& C=\text { est le résidu sec soluble (ppm) }
\end{aligned}
$$

\section{MATIÈRES EN SUSPENSION FORMÉES A PH 7}

(Voir Normes Industrielles Japonaises K0102 - 10.3) $7 \pm 0,5$.

Il s'agit des matières en suspension formées lorsque l'eau d'essai est neutralisée à un $\mathrm{pH}$ de

\section{Réactifs}

- $\quad$ Solution de $\mathrm{NaOH}$ (soude) (4 à $24 \mathrm{P} / \mathrm{V} \%$ )

- Acide acétique dilué dans de l'eau dans des proportions allant de $1 / 2$ à $1 / 6$, acide/eau. 


\section{$302 \mathrm{C}$}

page 22

\section{« Biodégradabilité dite Intrinsèque : Essai MITI Modifié (II) »}

\section{Mode opératoire}

Verser assez d'eau à examiner dans un bécher, pour obtenir plus de $5 \mathrm{mg}$ de matières en suspension et la neutraliser au moyen d'une solution de soude ou d'acide acétique dilué en fonction du caractère acide ou basique de l'eau d'essai. En neutralisant, veiller à ne pas trop augmenter la quantité de solution. Procéder ensuite, conformément aux indications qui précèdent, de manière à obtenir la quantité requise de matières en suspension et calculer la quantité. formée à pH 7 au moyen de la formule suivante :

$\mathrm{A}=\mathrm{B}-\mathrm{C}$

(voir plus haut pour l'explication des symboles).

\section{Remarques :}

(1) Selon le type d'eau d'égout, le poids de matières en suspension peut diminuer à la suite de la neutralisation. Dans ces conditions, le poids de matières en suspension doit être consigné comme matières en suspension formées à $\mathrm{pH} 7$.

(2) Les matières en suspension formées à $\mathrm{pH} 7$ peuvent être déterminées dans le liquide surnageant ou dans le filtrat après élimination des matières en suspension. Cette méthode s'applique à de l'eau à examiner contenant relativement peu de matières en suspension mais formant un précipité abondant après neutralisation (sans modifier la quantité de matières en suspension initiale). La méthode s'applique également à de l'eau usée formant relativement peu de précipité. Par contre, elle ne s'applique pas à de l'eau usée ayant tendance à former un complexe précipitable et à engendrer des réactions de dissolution lors de la neutralisation. 